\title{
A systematic review and meta-analysis of the effects of general anesthesia combined with continuous paravertebral block in breast cancer surgery and postoperative analgesia
}

\author{
Changsheng Feng, Duo Qian, Changlin Chen
}

Department of Anesthesiology, Affiliated Hospital of North Sichuan Medical College, Nanchong, China

Contributions: (I) Conception and design: C Feng, D Qian; (II) Administrative support: C Chen; (III) Provision of study materials or patients: C Feng, D Qian; (IV) Collection and assembly of data: All authors; (V) Data analysis and interpretation: C Feng, C Chen; (VI) Manuscript writing: All authors; (VII) Final approval of manuscript: All authors.

Correspondence to: Changsheng Feng. 63 Cultural Road, Shunqing District, Nanchong, China. Email: boxfchsh@126.com.

Background: This study aimed to compare the effects of general anesthesia (GA) combined with continuous paravertebral block (CPVB) in breast cancer surgery via systematic review and meta-analysis, in order to provide a theoretical basis for the clinical use of CPVB surgical analgesia.

Methods: A search of the PubMed, Embase, Medline, Ovid, Springer, and Web of Science databases was conducted to screen clinical trials on GA + CPVB for breast cancer surgery published before December 31, 2020. The Cochrane Handbook for Systematic Reviews of Intervention 5.0.2 was adopted for bias risk assessment, and Review Manager 5.3 software (RevMan, The Cochrane Collaboration, http://tech.cochrane. org/revman) was applied for meta-analysis of the literature.

Results: A total of 15 studies that satisfied the requirements were included, involving a total of 1,435 research subjects. The results of our meta-analysis showed the following: the visual analogue scale (VAS) score of the observation group (group A) was significantly reduced [mean difference (MD) $=-0.68 ; 95 \%$ confidential interval $(\mathrm{CI}):-1.04--0.33 ; \mathrm{Z}=3.80 ; \mathrm{P}=0.0001]$; the level of monocyte chemoattractant protein -1 (MCP-1) was notably decreased (MD =-18.64; 95\% CI: $-29.68--7.61 ; Z=3.31 ; P=0.0009)$; the level of tumor necrosis factor- $\alpha(\mathrm{TNF}-\alpha)$ was markedly lower $(\mathrm{MD}=-1.89 ; 95 \% \mathrm{CI}:-2.66--1.13 ; \mathrm{Z}=4.87$; $\mathrm{P}<0.00001$ ); the interleukin-6 (IL-6) level was obviously reduced (MD =-12.10; 95\% CI: $-19.22--4.99$; $\mathrm{Z}=3.33 ; \mathrm{P}=0.0009)$; and the incidence of postoperative adverse reactions was substantially decreased (MD $=0.16 ; 95 \%$ CI: 0.07-0.36; $\mathrm{Z}=4.47 ; \mathrm{P}<0.00001)$. Compared with group $\mathrm{B}$, the differences of the above five indicators showed statistical significance. In addition, the heart rate (HR) (MD =-1.56; 95\% CI: $-6.20-$ 3.08; $\mathrm{Z}=0.66 ; \mathrm{P}=0.51)$, mean arterial pressure (MAP) $(\mathrm{MD}=4.66 ; 95 \% \mathrm{CI}:-0.12-9.43 ; \mathrm{Z}=1.91 ; \mathrm{P}=0.06)$, Ramsay score ( $M D=0.44 ; 95 \% \mathrm{CI}:-0.06-0.93 ; \mathrm{Z}=1.73 ; \mathrm{P}=0.08)$ of patients in group A showed no statistical differences compared to group B.

Conclusions: GA + CPVB applied to breast cancer surgery for analgesia can reduce the levels of MCP1, TNF- $\alpha$, and IL- 6 in patients, thereby providing good postoperative analgesia. Therefore, GA + CPVB could effectively reduce the incidence of pain and adverse reactions in patients, and is effective for analgesia in breast cancer surgery.

Keywords: General anesthesia combined with continuous paravertebral block (GA combined with CPVB); breast cancer surgery; analgesia; meta-analysis

Submitted Mar 13, 2021. Accepted for publication May 19, 2021.

doi: $10.21037 /$ gs-21-272

View this article at: http://dx.doi.org/10.21037/gs-21-272 


\section{Introduction}

Breast cancer is one of the most common malignant tumors in women, accounting for approximately $15 \%$ of new cancers in China annually, and its incidence is increasing every year (1). At present, the early clinical treatment of breast cancer is still based on surgery, and gradually transitions to breast-conserving surgery. However, due to various stimuli, such as emotional stress, intraoperative trauma, and pain, surgery can easily lead to systemic inflammation, increasing the probability of postoperative complications (2). Pain is a common postoperative complication in breast cancer patients, with an incidence of approximately $25-60 \%$. Due to the impact of pain, patients are reluctant to perform deep breathing exercises after surgery, and there are residual secretions in the lungs, thereby limiting lung function. In addition, some symptoms such as nausea and vomiting seriously affect the quality of life of patients after surgery, leading to a significant increase in the length of hospitalization, medical expenses, and the probability of perioperative complications (3).

Breast-conserving surgery can be regarded as an enhanced recovery after surgery (ERAS) treatment method, which aims to improve the effectiveness of surgery, accelerate patient recovery, reduce the probability of postoperative complications, and improve the quality of life of patients (4). Analgesia has played an increasingly important role in ERAS. Good analgesia promotes the recovery of early postoperative activity and reduces the risk of postoperative complications (5). With regards to analgesia in breast cancer surgery, although traditional GA can meet the needs of the operation, the postoperative analgesic effect is poor. Thus, it is necessary to use large doses of opioid analgesic drugs in order to adequately satisfy the required postoperative analgesic effect, which may increase the risk of postoperative malignant vomiting, respiratory depression, and poor gastrointestinal activity (6). With the popularization of ultrasound visualization technology, analgesic technology has also been substantially developed. Research has found that local anesthetics can be injected into the paravertebral space under ultrasound guidance to block several segments of the patient's body and sympathetic nerves on the same side. The anesthesia technique is simple and easy to master, and the risk is low (7). Numerous studies have shown that thoracic paravertebral block (TPVB) can inhibit the unfavorable pathophysiological response of patients during the perioperative period, affect the intraoperative neuroendocrine stress response, decrease the use of analgesics, reduce postoperative pain, and improve breathing and circulation (8).

According to the needs of single or continuous block, TPVB can be divided into single-point TPVB (SPVB) and CPVB (9). At present, there have been a number of applications of GA + CPVB in the breast both domestically and internationally. There are related studies regarding cancer surgical analgesia, however the quality of the literature is uneven, and there is a lack of uniform treatment criteria. Therefore, we conducted a metaanalysis of the randomized control trials (RCTs) of GA + CPVB in the treatment of breast cancer, and indicators such as hemodynamics and postoperative analgesia in breast cancer patients were comprehensively evaluated. This study aimed to systematically evaluate and compare the safety and effectiveness of GA + CPVB in breast cancer surgery patients, in order to provide a reference for the analgesic methods of breast cancer surgery. We present the following article in accordance with the PRISMA reporting checklist (available at http://dx.doi. org/10.21037/gs-21-272).

\section{Methods}

\section{Inclusion and exclusion criteria}

The inclusion criteria were defined as follows: (I) the subjects of the study were patients who were clinically diagnosed with breast cancer, and were aged $\geq 18$ years; (II) the article was related to a RCT published in a foreign database in English; (III) the analgesia used in group A was GA + CPVB, and the surgical analgesic method applied in group B was GA; (IV) the baseline data of patients in groups A and B were comparable; (V) the dose and type of anesthetic drugs were not specified; and (VI) the evaluation indicators included the postoperative pain score, hemodynamics, and incidence of adverse reactions.

The exclusion criteria were defined as follows: (I) the article was a retrospective study, case report, cohort study, and other non-RCT research; (II) the subjects were nonbreast cancer patients or were animals or cells; (III) the documents were not published or provided in English; (IV) the anesthesia method utilized in group A was a SPVB or a trial combined without GA; (V) the research objects were breast cancer patients combined with other diseases; and (VI) the research data was incomplete and the corresponding effect index could not be calculated. 


\section{Literature search}

The literature search was performed in six English databases (PubMed, Embase, Medline, Ovid, Springer, and Web of Science) to screen for RCTs of breast surgery treated with GA + CPVB published before December 31, 2020. The keywords for the search included "breast cancer surgery", "Breast cancer", "general anesthesia", "thoracic paravertebral nerve block", "TPVB", "continuous thoracic paravertebral nerve block", "CPVB", and "breast", which were connected with "and" or "or" for joint searches. The search was performed by two researchers using independent search methods.

\section{Literature screening}

After the search, NoteExpress 3.2 (http://www.inoteexpress. com/aegean/) was used to establish a document database, which was checked for duplicate documents that were subsequently eliminated. The two researchers manually screened the remaining documents. They were required to initially read the titles and abstracts of the retrieved documents, eliminate documents that obviously did not meet the inclusion criteria, and then complete literature reading according to the document inclusion and exclusion criteria, so as to determine whether to include the literature in the meta-analysis. In the process of document screening, any differences of opinion between the two researchers were resolved through discussion and consensus. If a consensus could not be reached, a third party was invited to make a decision after arbitration.

\section{Information extraction}

Two researchers were required to draw data record tables based on the basic information of the literature, the characteristics of the research objects, intervention measures, outcome indicators, and bias evaluation. Two researchers independently pre-extracted data from documents that met the inclusion criteria. The extraction was completed and cross-examined, and disagreements were resolved by discussion; otherwise, an agreement was decided after third party arbitration. The data extracted from the literature mainly included: title; first author (only one name); publication time; research area; ages of the research objects; size of the research samples; comparability of the baseline; design and implementation methods; intervention and control measures; anti-bias measures; research outcome indicators; and research data.

\section{Quality assessment of documents}

The risk-of-bias assessment criteria provided in the Cochrane Handbook for Systematic Reviews of Intervention 5.0.2 were applied to evaluate the risk-of-bias included in the original literature. The included studies were evaluated according to the generation of random sequence, implementation of patients and evaluator blinding method, implementation of outcome evaluator blinding method, completion of the research data, available selective report results, and the sources of bias. Any differences between the two researchers were resolved through discussion of the different opinions. If a consensus could not be reached, a third party was invited to make a decision after arbitration.

\section{Statistical methods}

The risk-of-bias of the included studies was evaluated using the Cochrane Handbook for Systematic Reviews of Interventions 5.0.2 software. STATA 11.0 software (https:// www.stata.com) was adopted to merge the statistics of the included studies, and Review Manager 5.3 software (RevMan, The Cochrane Collaboration, http://tech. cochrane.org/revman) was employed for meta-analysis of the merged statistics and drawing of the forest and funnel diagrams. Relative risk (RR) was defined as the effect size of the binary variables in the count data, such as postoperative adverse reactions, and the $95 \%$ CI was calculated. For continuous variables in the measurement data, such as HR, MAP, and VAS, the weighted MD was considered as the effect size if the units of the detection indicators were the same; and the standardized MD (SMD) was considered as the effect size if the units of the detection indicators were not the same. If the research results of various literatures could be combined, a meta-analysis was required. The $\mathrm{I}^{2}$ test was utilized to evaluate the heterogeneity of the included literature, with larger $\mathrm{I}^{2}$ values indicating greater heterogeneity. If $\mathrm{I}^{2}>50 \%$ and the source of heterogeneity could not be explained, a random effects models (REM) combined effect size was used for meta-analysis. However, $\mathrm{I}^{2}<50 \%$ meant that the literature heterogeneity was good, and a fixed effect model (FEM) combined effect size was adopted for meta-analysis. If the research data was less than two items (meaning that meta-analysis was impossible), a descriptive analysis could be adopted. The combined effect size was tested using the U-test and 95\% CI. The 
$\mathrm{U}$-test result was expressed as a $\mathrm{P}$ value, and $\mathrm{P}<0.05$ was considered to indicate a statistically significant difference. Binary variables were tested with $95 \%$ CI. A $95 \%$ CI $>1$ or $<1$ indicated that the difference was statistically significant; when the $95 \%$ CI contained 1, the difference was not statistically obvious. Continuous variables were tested with $95 \% \mathrm{CI}$; when the $95 \% \mathrm{CI}$ was $>0$ or $<0$, the difference was statistically meaningful.

\section{Results}

\section{Literature search results}

A preliminary search of six English databases revealed a total of 1,102 related documents, including 308 documents from the PubMed database, 215 documents from the Embase database, 216 documents from the Medline database, 143 documents from the Springer database, 105 documents from the Ovid database, and 115 articles from the Web of Science database. Following the preliminary search, all 1,102 literature titles were imported into NoteExpress 3.2 , and 829 literatures remained after rechecking and culling. Two researchers were allowed to read the titles and abstracts of the remaining literature, and select those that satisfied the inclusion and exclusion criteria. After this process, 86 documents remained. Next, the two researchers were allowed to read and cross-examine the text of the literature, and then screen and exclude literature based on the inclusion and exclusion criteria. Finally, a total of 15 studies were included in this meta-analysis, as shown in Table 1. All of which were publicly published RCTs between 2005 to 2020, involving 1,435 subjects aged 18-73 years. The baseline data, such as the age of group A and group B patients, were comparable. The literature search procedure is displayed in Figure 1.

\section{Bias risk assessment of included studies}

The Cochrane Handbook for Systematic Reviews of Interventions 5.0.2 software was used to evaluate the risk-of-bias of the 15 RCTs included in this study, and the Review Manager 5.3 software was used to output the bias risk diagrams. The risk-of-bias assessment included six aspects. (I) Generation of random sequence: the 15 RCTs all reported the use of randomization, of which 12 $(11,13,14-20,22-24)$ utilized a random number table as the specific randomization method, suggesting that these 12 RCTs were low risk. Meanwhile, the remaining three
RCTs $(10,12,21)$ only mentioned random grouping, but failed to specifically describe which random method was used, indicating that their risk-of-bias was unclear. (II) Allocation concealment: none of the 15 documents (10-24) mentioned whether or not "allocation concealment" was performed, indicating that their risk-of-bias was unclear. (III) Implementation of patient blinding method: one RCT (17) clearly mentioned blinding of subjects (low risk); 12 documents (10-14,16,18-20,22-24) mentioned "Patients had known and signed the informed consent forms", indicating that they were high risk; and two documents $(15,21)$ failed to mention whether the blinding method was used, indicating that their risk-of-bias was unclear. (IV) Implementation of outcome evaluator blinding method: none of the 15 RCTs (10-24) mentioned whether the outcome evaluator was blinded, suggesting that the riskof-bias was unclear. (V) Completion of the research data: the outcome data of all 15 RCTs (10-24) were complete, suggesting low risk. (VI) Selective report: none of the 15 RCTs (10-24) had selective report, indicating low risk (7). Other risks of bias: two RCTs $(11,20)$ had inconsistent numbers of patients in group A and group B, suggesting high risk. The remaining 13 documents (10,12-19,21-24) exhibited an unclear risk since the other biases could not be determined. The risk-of-bias assessment results are shown in Figures 2,3.

\section{Heart rate}

A total of seven RCTs $(10-13,16,23,24)$ analyzed the postoperative HR of patients, including 685 breast cancer patients, with 343 cases in group A and 342 cases in group B. According to the results of the heterogeneity test, there was a certain degree of heterogeneity among the included studies $\left(\mathrm{I}^{2}=98 \%\right.$ and $\left.\mathrm{P}<0.00001\right)$, and thus, the analysis was performed using a REM. The results showed that the combined effect of meta-analysis was $\mathrm{MD}=-1.56 ; 95 \% \mathrm{CI}$ : -6.20-3.08]; $\mathrm{Z}=0.66$; and $\mathrm{P}=0.51$ (Figure 4), which suggested that patients who received GA + CPVB in breast cancer surgery exhibited no significant difference in HR compared with those who received conventional GA.

\section{Mean arterial pressure}

Seven RCTs $(11-13,16,18,23,24)$ analyzed the postoperative MAP of patients, and a total of 593 breast cancer patients were included, including 297 cases in group A and 296 cases in group B. According to the results of the heterogeneity 
Table 1 Basic characteristics of literature included in this study



GA, general anesthesia (GA); CPVB, continuous paravertebral block.

test, there was a certain degree of heterogeneity among the included studies $\left(\mathrm{I}^{2}=94 \%\right.$ and $\left.\mathrm{P}<0.00001\right)$, and therefore, the analysis was performed using a REM. The results showed that the combined effect of meta-analysis was MD
$=4.66 ; 95 \%$ CI: -0.12 to $9.43 ; \mathrm{Z}=1.91$; and $\mathrm{P}=0.06$ (Figure 5), which suggested that patients who received GA + CPVB analgesia showed no significant difference in postoperative MAP compared to those who received conventional GA. 


\section{VAS score}

Eleven of the 15 RCTs documents analyzed the postoperative VAS scores of patients (10-14,17-19,21-23), and 977 breast cancer patients were included, with 489 cases in group A and 488 cases in group B. The heterogeneity test results revealed that there was a certain degree of heterogeneity among the included studies $\left(\mathrm{I}^{2}=97 \%\right.$ and $\mathrm{P}<0.00001)$, and thus, the analysis was conducted using a REM. The results indicated that the combined effect of meta-analysis was $\mathrm{MD}=-0.68 ; 95 \% \mathrm{CI}:-1.04$ to -0.33 ; $\mathrm{Z}=3.80$; and $\mathrm{P}=0.0001$ (Figure 6), indicating that patients

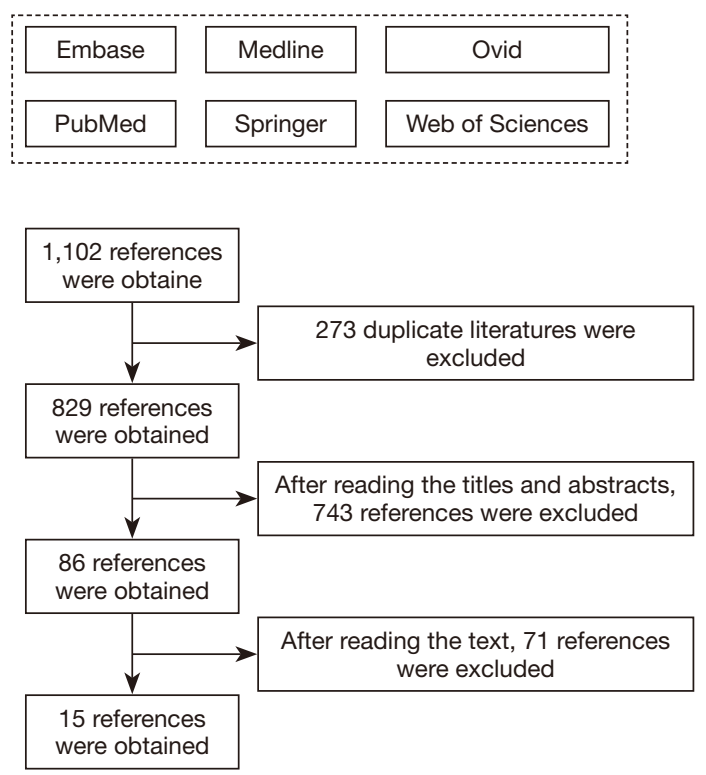

Figure 1 Literature search process. who received GA + CPVB showed a significant difference in the VAS scores compared to those who received conventional GA. These results demonstrate that GA + CPVB had a better postoperative analgesic effect.

\section{Ramsay score}

A total of three RCTs $(10,12,23)$ included analyzed the postoperative Ramsay scores of patients, and 390 breast cancer patients were included, including 195 cases in group A and 195 cases in group B. According to the results of the heterogeneity test, heterogeneity was observed among the included studies ( $\mathrm{I}^{2}=96 \%$ and $\left.\mathrm{P}<0.00001\right)$; hence, a REM was used for analysis. As shown in Figure 7, the combined effect of meta-analysis was $\mathrm{MD}=0.44 ; 95 \% \mathrm{CI}$ : -0.06 to $0.93 ; \mathrm{Z}=1.73$; and $\mathrm{P}=0.08$, which suggested that patients who received GA + CPVB analgesia showed no statistically observable difference in the postoperative Ramsay scores compared to those who received conventional GA.

\section{Monocyte Chemoattractant Protein -1}

Three RCTs (13-15) analyzed the levels of MCP-1 in patients after surgery. A total of 284 breast cancer patients were included, including 142 in group A and 142 in group B. The heterogeneity test results found heterogeneity among the included studies $\left(\mathrm{I}^{2}=98 \%\right.$ and $\left.\mathrm{P}<0.00001\right)$, and thus, a REM was adopted for analysis. The results indicated that the combined effect of meta-analysis was $\mathrm{MD}=-18.64 ; 95 \%$ CI: -29.68 to $-7.61 ; Z=3.31$; and $\mathrm{P}=0.0009$ (Figure 8 ), which demonstrated that patients who received GA + CPVB

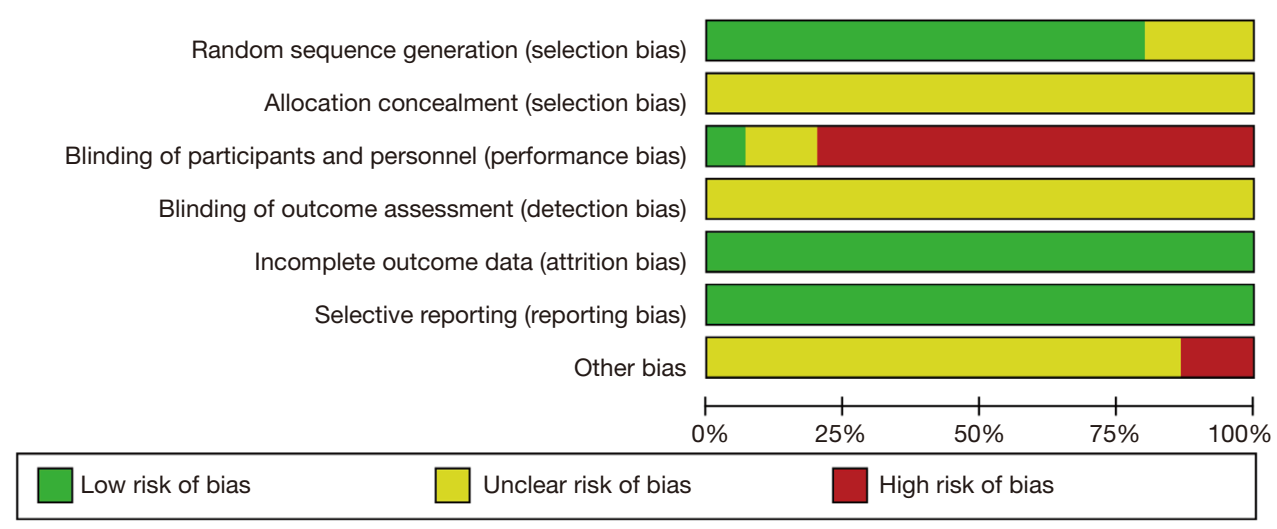

Figure 2 Bar graph showing the bias assessment of the included literature. 


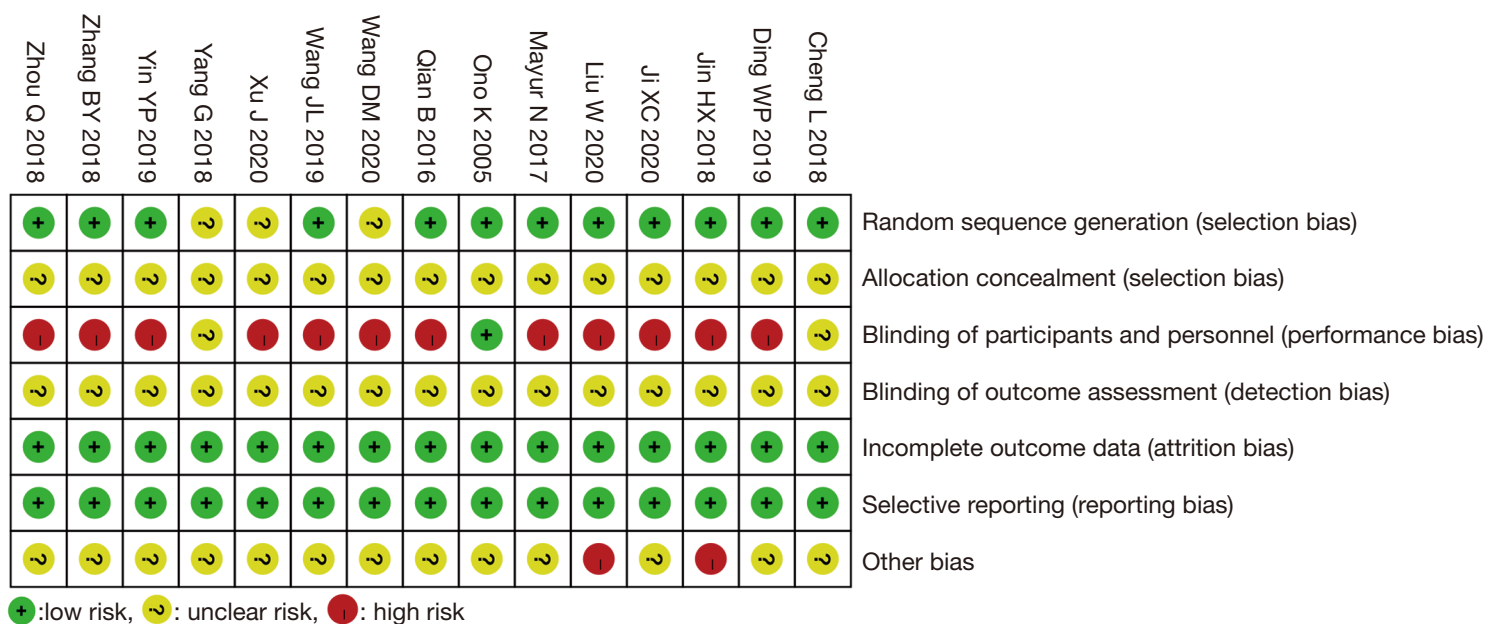

Figure 3 Results of bias assessment of included literature.

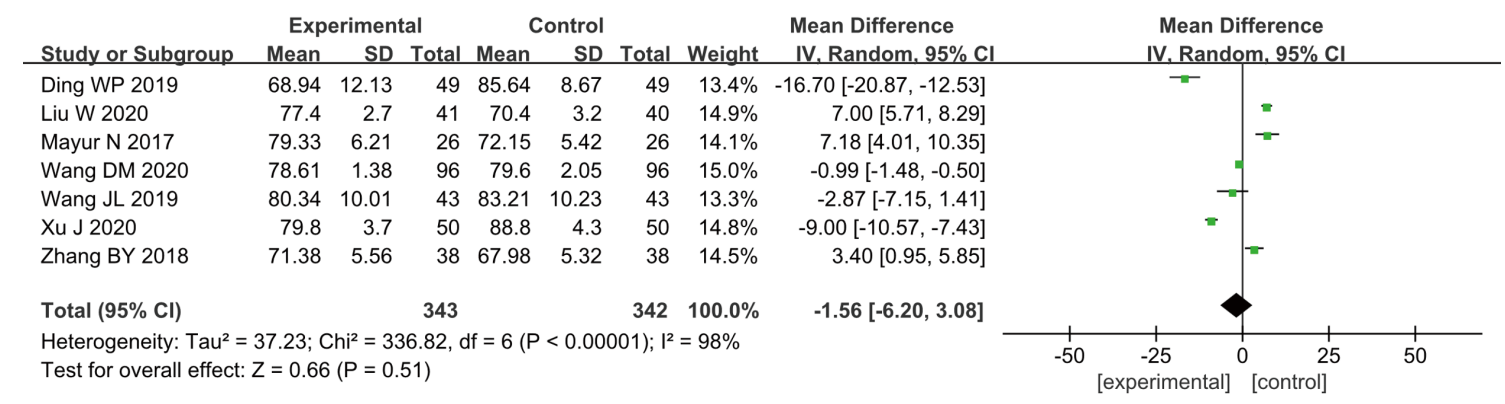

Figure 4 The forest diagram for the postoperative HR of the two groups of patients.

\begin{tabular}{|c|c|c|c|c|c|c|c|c|c|c|c|}
\hline \multirow[b]{2}{*}{ Study or Subgroup } & \multicolumn{3}{|c|}{ Experimental } & \multicolumn{2}{|c|}{ Control } & \multirow[b]{2}{*}{ Total } & \multirow[b]{2}{*}{ Weight } & \multirow{2}{*}{$\begin{array}{l}\text { Mean Difference } \\
\text { IV. Random. } 95 \% \mathrm{Cl}\end{array}$} & \multirow{2}{*}{\multicolumn{3}{|c|}{$\begin{array}{c}\text { Mean Difference } \\
\text { IV. Random. } 95 \% \mathrm{CI}\end{array}$}} \\
\hline & Mean & SD & Total & Mean & SD & & & & & & \\
\hline Ding WP 2019 & 93.51 & 9.64 & 49 & 103.21 & 12.64 & 49 & $13.6 \%$ & $-9.70[-14.15,-5.25]$ & $\square$ & & \\
\hline Liu W 2020 & 112.2 & 5.7 & 41 & 97.4 & 5.8 & 40 & $14.8 \%$ & $14.80[12.30,17.30]$ & & $=$ & \\
\hline Mayur N 2017 & 109.38 & 8.46 & 26 & 100.35 & 6.89 & 26 & $13.8 \%$ & $9.03[4.84,13.22]$ & & - & \\
\hline Wang JL 2019 & 101.45 & 6.79 & 50 & 98.26 & 7.64 & 50 & $14.6 \%$ & $3.19[0.36,6.02]$ & & - & \\
\hline Xu J 2020 & 94.6 & 4.5 & 50 & 90.6 & 4.6 & 50 & $15.1 \%$ & $4.00[2.22,5.78]$ & & $=$ & \\
\hline Yin YP 2019 & 118.25 & 10.32 & 43 & 113.36 & 11.12 & 43 & $13.5 \%$ & $4.89[0.36,9.42]$ & & - & \\
\hline Zhang BY 2018 & 100.58 & 7.01 & 38 & 95.03 & 6.67 & 38 & $14.5 \%$ & $5.55[2.47,8.63]$ & & $\pi$ & \\
\hline Total $(95 \% \mathrm{Cl})$ & & & 297 & & & 296 & $100.0 \%$ & $4.66[-0.12,9.43]$ & & & \\
\hline $\begin{array}{l}\text { Heterogeneity: } \mathrm{Tau}^{2}= \\
\text { Test for overall effect }\end{array}$ & $\begin{array}{l}38.47 ; \mathrm{Ch} \\
Z=1.91\end{array}$ & $\begin{array}{l}\mathrm{i}^{2}=105 \\
(\mathrm{P}=0.0\end{array}$ & 6) & $=6(\mathrm{P}$ & 0.0000 & $;\left.\right|^{2}=$ & $94 \%$ & & $\begin{array}{ll}-50 & -25 \\
\text { [experimental] }\end{array}$ & $\begin{array}{lr}0 & 25 \\
\text { [control] }\end{array}$ & 50 \\
\hline
\end{tabular}

Figure 5 The forest diagram for the postoperative mean arterial pressure (MAP) of the two groups of patients.

showed obvious difference in postoperative MCP-1 levels compared to those who received conventional GA.

\section{Tumor necrosis factor $-\alpha(T N F-\alpha)$}

Five RCTs $(14-16,19,21)$ analyzed the postoperative TNF- $\alpha$ levels of patients. In total, 426 breast cancer patients were enrolled, including 213 cases in group A and 213 cases in group B. According to the results of the heterogeneity test, it was found that there was a certain degree of heterogeneity among the included studies $\left(\mathrm{I}^{2}=91 \%\right.$ and $\left.\mathrm{P}<0.00001\right)$, and thus, the analysis was performed using a REM. The results 


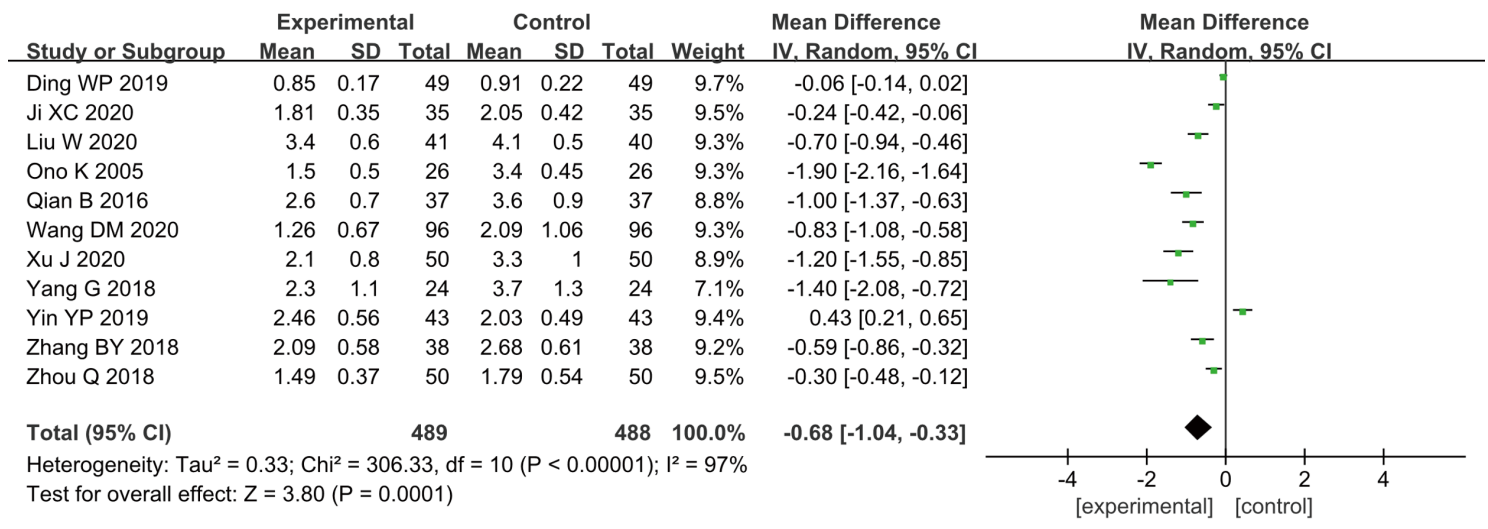

Figure 6 The forest diagram for postoperative visual analogue scale (VAS) scores of the two groups of patients.

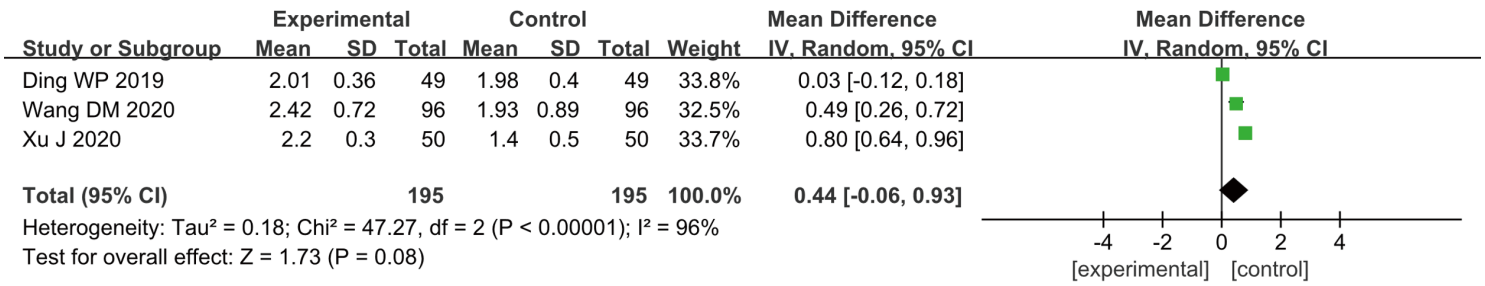

Figure 7 The forest diagram for postoperative Ramsay score of the two groups of patients.

\begin{tabular}{|c|c|c|c|c|c|c|c|c|c|}
\hline \multirow[b]{2}{*}{ Study or Subgroup } & \multicolumn{3}{|c|}{ Experimental } & \multicolumn{3}{|c|}{ Control } & \multicolumn{2}{|r|}{ Mean Difference } & \multirow{2}{*}{$\begin{array}{c}\text { Mean Difference } \\
\text { IV. Random, } 95 \% \mathrm{Cl}\end{array}$} \\
\hline & Mean & SD & Total & Mean & SD & Total & Weight & IV. Random, $95 \% \mathrm{Cl}$ & \\
\hline Zhou Q 2018 & 40.2 & 4.68 & 50 & 64.1 & 7.7 & 50 & $33.3 \%$ & $-23.90[-26.40,-21.40]$ & - \\
\hline Zhang BY 2018 & 19.01 & 4.64 & 38 & 26.06 & 6.71 & 38 & $33.3 \%$ & $-7.05[-9.64,-4.46]$ & 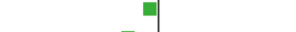 \\
\hline Cheng L 2018 & 40.13 & 4.75 & 54 & 65.07 & 6.82 & 54 & $33.4 \%$ & $-24.94[-27.16,-22.72]$ & - \\
\hline Total $(95 \% \mathrm{Cl})$ & & & 142 & & & 142 & $100.0 \%$ & $-18.64[-29.68,-7.61]$ & \\
\hline $\begin{array}{l}\text { Heterogeneity: } \mathrm{Tau}^{2}= \\
\text { Test for overall effect }\end{array}$ & $\begin{array}{l}3.51 ; C \\
=3.31\end{array}$ & $\begin{array}{l}\mathrm{hi}^{2}=1 \\
(\mathrm{P}=0\end{array}$ & $\begin{array}{l}23.55 \\
0009)\end{array}$ & $\mathrm{df}=2$ & $<0$. & 0001); & $I^{2}=98 \%$ & & $\begin{array}{ccccc}-100 & -50 & 0 & 50 & 100 \\
\text { [experimental] } & \text { [control] }\end{array}$ \\
\hline
\end{tabular}

Figure 8 The forest diagram for postoperative monocyte chemoattractant protein -1 (MCP-1) levels of the two groups of patients.

showed that the combined effect of meta-analysis was $\mathrm{MD}$ $=-1.89 ; 95 \%$ CI: -2.66 to $-1.13 ; Z=4.87$; and $\mathrm{P}<0.00001$ (Figure 9), which indicated that GA + CPVB could lower the postoperative levels of TNF- $\alpha$ in breast cancer patients, exhibiting a significant statistical difference compared to conventional GA.

\section{Interleukin-6}

Five RCTs $(13,15,16,19,21)$ analyzed postoperative IL-6 levels, and 402 breast cancer patients were included, with 201 cases in group A and 201 cases in group B. The results of the heterogeneity test found that there was a certain degree of heterogeneity among the included studies $\left(\mathrm{I}^{2}=97 \%\right.$ and $\mathrm{P}<0.00001)$, so a REM was used for analysis. The results showed that the combined effect of meta-analysis was $M D=-12.10 ; 95 \% C I:-19.22$ to $-4.99 ; Z=3.33$; and $\mathrm{P}=0.0009$ (Figure 10), indicating that the levels of IL-6 in patients who received GA + CPVB in breast cancer surgery was significantly different from those in patients who received conventional GA.

\section{Postoperative adverse reactions}

Six RCTs $(10,12,13,18,20,23)$ analyzed the adverse reactions of patients after surgery, and 750 breast cancer patients 


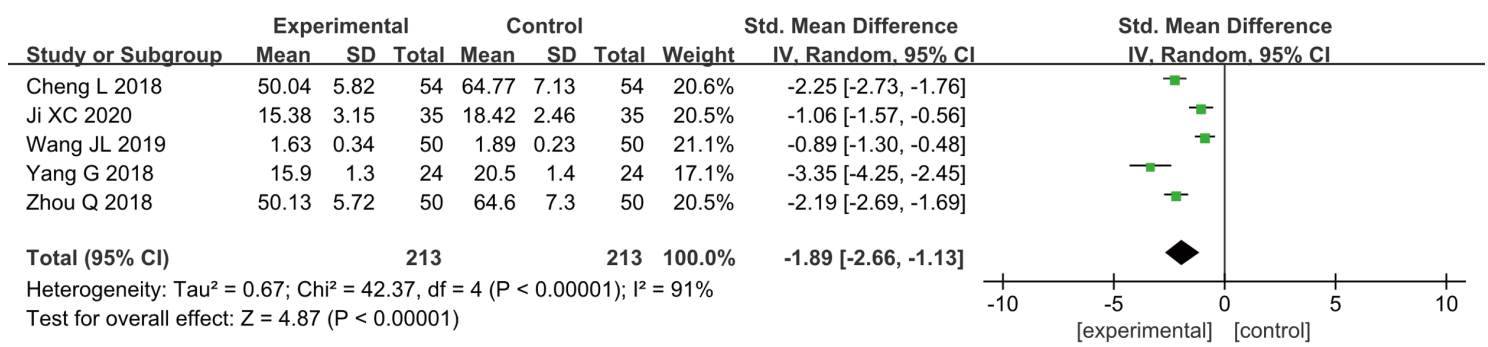

Figure 9 The forest diagram for postoperative tumor necrosis factor- $\alpha(\mathrm{TNF}-\alpha)$ levels of the two groups of patients.

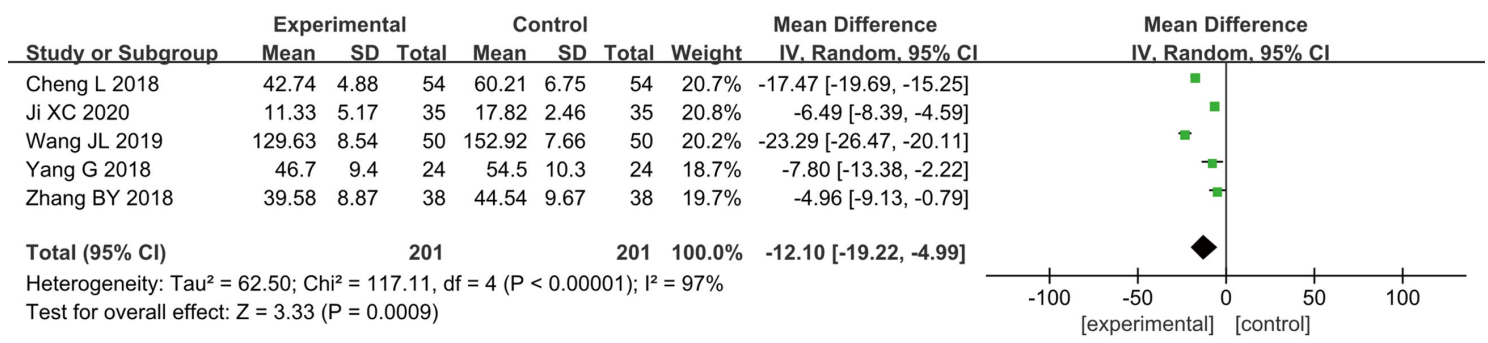

Figure 10 The forest diagram for postoperative interleukin-6 (IL-6) levels of the two groups of patients.

\begin{tabular}{|c|c|c|c|c|c|c|c|c|c|c|}
\hline Study or Subgroup & \multicolumn{2}{|c|}{ Experimental } & \multicolumn{2}{|c|}{ Control } & & Odds Ratio & \multicolumn{4}{|c|}{$\begin{array}{c}\text { Odds Ratio } \\
\text { M-H, Random, } 95 \% \mathrm{Cl}\end{array}$} \\
\hline Ding WP 2019 & 1 & 49 & 11 & 49 & $97 \%$ & $007[0010581$ & & & & \\
\hline Jin HX 2018 & 3 & 102 & 49 & 96 & $172 \%$ & $0.03\left[\begin{array}{lll}0.01 & 0 & 10\end{array}\right]$ & & & & \\
\hline Wang DM 2020 & 15 & 96 & 36 & 96 & $23.5 \%$ & $0.31[0.16,0.61]$ & & & & \\
\hline Xu J 2020 & 4 & 50 & 14 & 50 & $17.4 \%$ & $0.22[0.07,0.74]$ & & & & \\
\hline Yin YP 2019 & 3 & 43 & 10 & 43 & $15.5 \%$ & $0.25[0.06,0.97]$ & & & & \\
\hline Zhang BY 2018 & 4 & 38 & 11 & 38 & $16.8 \%$ & $0.29[0.08,1.01]$ & & & & \\
\hline Total $(95 \% \mathrm{Cl})$ & & 378 & & 372 & $100.0 \%$ & $0.16[0.07,0.36]$ & & & & \\
\hline Total events & 30 & & 131 & & & & & & & \\
\hline $\begin{array}{l}\text { Heterogeneity: } \mathrm{Tau}^{2} \\
\text { Test for overall effect }\end{array}$ & $\begin{array}{l}.58 ; \mathrm{Chi}^{2} \\
=4.47(\mathrm{~F}\end{array}$ & $\begin{array}{l}13.14 \\
0.00\end{array}$ & $\begin{array}{l}d f=5(P \\
01)\end{array}$ & $=0.02$ & $\mathrm{I}^{2}=62 \%$ & & 0.001 & $\begin{array}{c}0.1 \\
\text { [experimental] }\end{array}$ & $\begin{array}{rr}1 & 10 \\
\text { [control] }\end{array}$ & 1000 \\
\hline
\end{tabular}

Figure 11 The forest diagram for postoperative adverse reactions of the two groups of patients.

were included, with 378 cases in group A and 372 cases in group B. According to the heterogeneity test results, a certain degree of heterogeneity was found among the included studies $\left(\mathrm{I}^{2}=62 \%\right.$ and $\left.\mathrm{P}=0.02\right)$, and hence, a REM was employed for analysis. The results showed that the combined effect of meta-analysis was $\mathrm{MD}=0.16 ; 95 \% \mathrm{CI}$ : 0.07-0.36; $\mathrm{Z}=4.47$; and $\mathrm{P}<0.00001$ ) (Figure 11), indicating that the incidence of adverse reactions for patients who received GA + CPVB analgesia was significantly different compared to patients who received conventional GA. GA + CPVB analgesia could reduce the probability of adverse reactions after breast cancer surgery.

\section{Analysis of publication bias}

Using Review Manager 5.3 software, the publication bias of the results and postoperative analgesia indicators of patients who received GA + CPVB in breast cancer surgery were analyzed (Figure 12). It was clear that the MAP and incidence of postoperative adverse reactions of the patients were basically distributed within the CI, showing low bias. Some spots in the funnel charts of HR, VAS score, Ramsay score, MCP-1, TNF- $\alpha$, and IL-6 of the patients were scattered outside the CI with relatively scattered distribution, indicating that there was a certain publication 
A

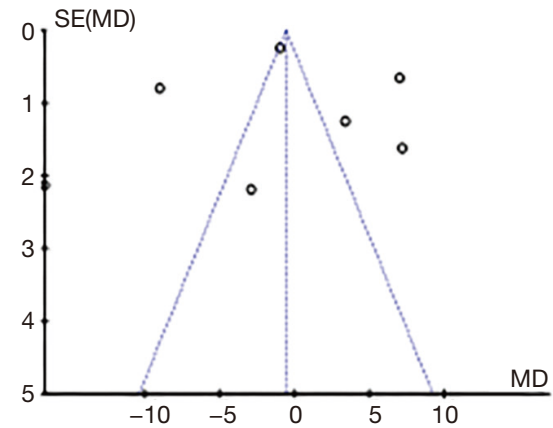

C

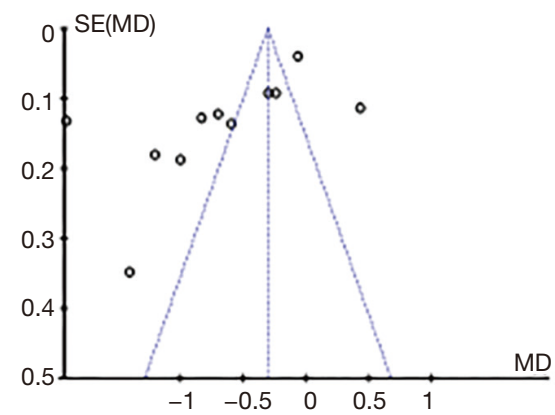

$\mathrm{E}$

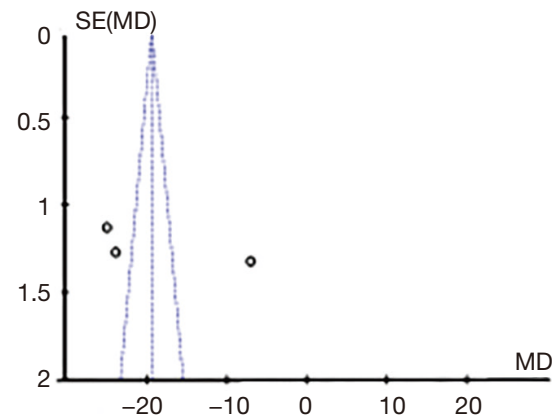

G

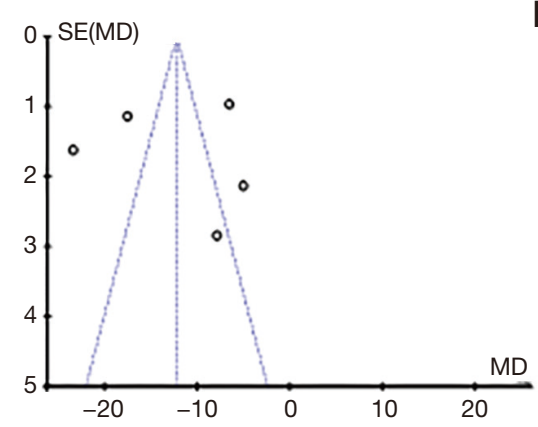

B

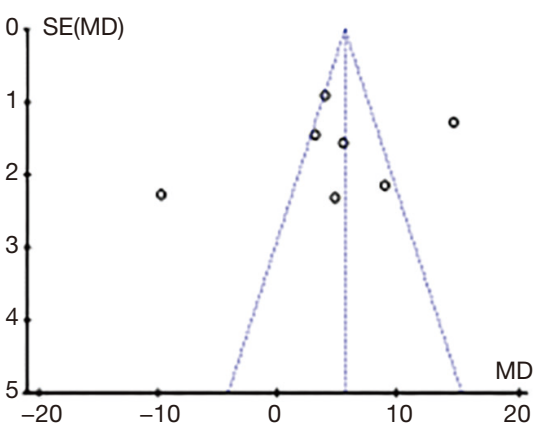

D

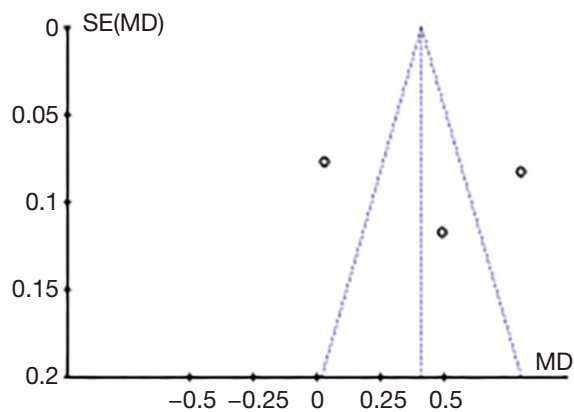

$\mathrm{F}$

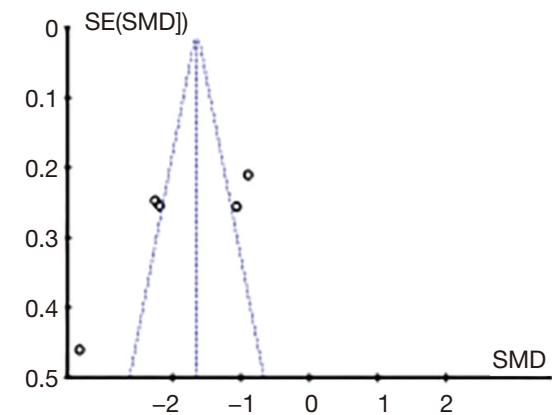

$\mathrm{H} \quad 0$ S SE(log[OR])

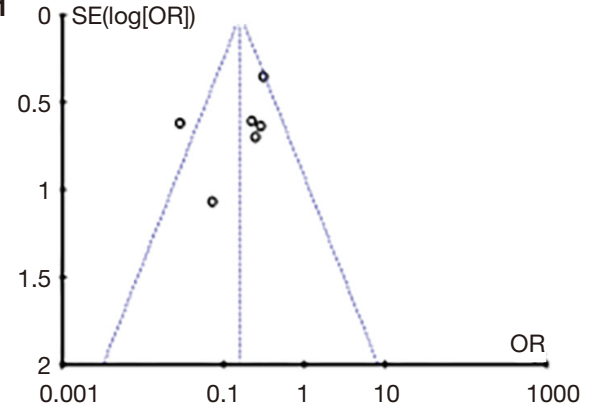

Figure 12 The funnel chart of each evaluation indicator. A, B, C, D, E, F, G, and H referred to the evaluation results of heart rate (HR), mean arterial pressure (MAP), visual analogue scale (VAS) score, Ramsay score, monocyte chemoattractant protein 1 (MCP-1), tumor necrosis factor- $\alpha(\mathrm{TNF}-\alpha)$, interleukin-6 (IL-6), and postoperative adverse reactions, respectively. 
bias in the included literature.

\section{Discussion}

Breast cancer surgery is more common in middle-aged and elderly women, and will typically result in considerable trauma to the patient. The rate of postoperative pain in patient is relatively high. The invasive surgical treatment will lead to postoperative stress and inflammatory responses, and hinder the postoperative recovery of patients (25). Conventional GA is commonly used in breast cancer surgery, mainly with opioids for analgesia. However, the drug dose is high, and only has an effect on the limbic system of the cerebral cortex and the projection system of the hypothalamus to the cortex, causing the patient to respond after awakening. The irritation is strong and the postoperative pain is substantial (26). With the development of medical technology, regional nerve block has become an important means of clinical auxiliary analgesia. In addition, the safety of regional nerve block has been significantly improved with the help of ultrasound guidance. CPVB technology effectively controls painful stimulation as well as the impact on hemodynamics, and is considered by scholars to be a safe and efficient analgesic technology (27).

The results of this study showed that there was no obvious difference in the postoperative HR and MAP for patients in groups A and B. However, it was impossible to prove that GA + CPVB can reduce HR and increase MAP. Comparison of the postoperative VAS scores of the two groups of patients revealed that the VAS scores of patients in group A were significantly lower than those of group B, suggesting that GA + CPVB had a better analgesic effect. A possible reason for this may be that the anesthesia injected by CPVB blocks the spinal nerves of the intervertebral foramen, improves the analgesic effect of the ipsilateral body, and provides patients with better perioperative analgesia. Also, the levels of MCP-1, TNF- $\alpha$, and IL-6 were markedly lower in group B compared to those in group A. A possible explanation for this may be that GA + CPVB completely blocks the process of traumatic stimulation from the peripheral surgery to the central nervous system. Meanwhile, GA only has an inhibitory effect on the hypothalamus or cerebral cortex, resulting in central neuritis in patients due to strong surgical stimulation. Moreover, the incidence of adverse reactions was notably lower in group A compared to group B, which may be due to the increased incidence of adverse reactions, such as nausea and vomiting, lethargy, and urinary retention in group B patients during analgesia. However, the GA + CPVB analgesia used in group A reduced the dose of opioid analgesics.

\section{Conclusions}

This meta-analysis examined 15 RCTs (involving 1,435 breast cancer patients) to investigate the effect of GA + CPVB on breast cancer surgery. The results showed that compared with conventional GA, GA + CPVB can greatly reduce the VAS score, MCP-1, TNF- $\alpha$, and IL-6 levels, as well as the incidence of postoperative adverse reactions in patients, but had no obvious impacts on HR, MAP, and the Ramsay score.

There were some limitations in this study that should be noted. Firstly, there was a considerable publication bias in some of the included studies. Additionally, due to the differences in the research direction of the authors of the included RCTs, some analysis indicators contained a small number of samples, and the meta-analysis results were not accurate enough. Therefore, it is necessary to select larger samples and high-quality RCTs to verify the effect of GA + CPVB for breast cancer surgery in future studies.

\section{Acknowledgments}

Funding: None.

\section{Footnote}

Reporting Checklist: The authors have completed the PRISMA reporting checklist. Available at http://dx.doi. org/10.21037/gs-21-272

Conflicts of Interest: All authors have completed the ICMJE uniform disclosure form (available at http://dx.doi. org/10.21037/gs-21-272). The authors have no conflicts of interest to declare.

Ethical Statement: The authors are accountable for all aspects of the work in ensuring that questions related to the accuracy or integrity of any part of the work are appropriately investigated and resolved.

Open Access Statement: This is an Open Access article distributed in accordance with the Creative Commons Attribution-NonCommercial-NoDerivs 4.0 International License (CC BY-NC-ND 4.0), which permits the noncommercial replication and distribution of the article with 
the strict proviso that no changes or edits are made and the original work is properly cited (including links to both the formal publication through the relevant DOI and the license). See: https://creativecommons.org/licenses/by-nc$\mathrm{nd} / 4.0 \%$.

\section{References}

1. Zhang YM, Liu YJ. A review for the surgical management of breast cancer and the latest developments. Journal of Modern Oncology 2015;23:719-22.

2. Pérez-González O, Cuéllar-Guzmán LF, Soliz J, Cata JP. Impact of Regional Anesthesia on Recurrence, Metastasis, and Immune Response in Breast Cancer Surgery: A Systematic Review of the Literature. Reg Anesth Pain Med 2017;42:751-6.

3. Cheng GS, Ilfeld BM. A review of postoperative analgesia for breast cancer surgery. Pain Manag 2016;6:603-18.

4. Temple-Oberle C, Shea-Budgell MA, Tan M, et al. Consensus Review of Optimal Perioperative Care in Breast Reconstruction: Enhanced Recovery after Surgery (ERAS) Society Recommendations. Plast Reconstr Surg 2017;139:1056e-71e.

5. Shi XQ, Deng DL. The application of ultrasound guided continuous paravertebral nerve block in postoperative analgesia of breast cancer patients. Journal of Snake 2018;30:218-20.

6. Sessler DI, Pei L, Huang Y, et al. Recurrence of breast cancer after regional or general anaesthesia: a randomised controlled trial. Lancet 2019;394:1807-15.

7. Martsiniv VV, Loskutov OA, Strokan AM, et al. Efficacy of pectoral nerve block type II versus thoracic paravertebral block for analgesia in breast cancer surgery. Wiad Lek 2020;73:1470-5.

8. Pawa A, Wight J, Onwochei DN, et al. Combined thoracic paravertebral and pectoral nerve blocks for breast surgery under sedation: a prospective observational case series. Anaesthesia 2018;73:438-43.

9. Zhang W, Li J, Geng QT, et al. Investigation on thoracic paravertebral block by single injection or multiple injections in patients with video-assisted thoracoscopic lung lobe surgery. Journal of Clinical Anesthesiology 2014;30:109-13.

10. Wang DM, Xu L, Miao HM, et al. Ultrasound-assisted Continuous Thoracic Paravertebral Nerve Block Combined with General Anesthesia for the Anesthesia and Postoperative Analgesia of Radical Mastectomy of Breast Cancer. Medical \& Pharmaceutical Journal of Chinese
People's Liberation Army 2020;32:117-20.

11. Liu W, Li WB, Zhao L, et al. Application of Laryngeal Mask General Anesthesia Combined with Ultrasound -guided Parathoracic Nerve Block in Anesthesia and Postoperative Analgesia for Radical Mastectomy of Breast Cancer. China Foreign Medical Treatment 2020;39:59-61.

12. Xu J, Yao HL, He CY, et al. Effects of ultrasound-guided thoracic paravertebral nerve block anesthesia combined with general anesthesia on elderly patients with breast cancer. Medical Journal of Chinese People's Health 2020;32:118-120, 123.

13. Zhang BY, Xiao JB. The effect of ultrasound-guided pleural paravertebral block combined with general anesthesia on serum monocyte chemokinin-1, interleukin-6 and interleukin-10 levels in patients with early breast cancer after modified radical mastectomy. Journal of Hainan Medical University 2018;24:1683-7.

14. Zhou Q, Xie M, Huang JX. Effect of Ultrasound-guided Thoracic Paraspinal Block Combined with General Anesthesia on the Changes of Serum Prostaglandin E2 and Monocyte Chemoattractant Protein-1 Levels in Patients with Early Breast Cancer after Modified Radical Mastectomy. Sichuan Medical Journal 2018;39:1040-4.

15. Cheng L, Tu XL. Effects of ultrasound-guided thoracic paravertebral block combined with general anesthesia on the secretion of pain-related mediators after modified radical mastectomy. Journal of Hainan Medical University 2018;24:45-8.

16. Wang JL, Xu GH. The correlations of the changes of T-lymphocyte subset counts with postoperative infection and cognitive dysfunction in patients with breast cancer after surgery by ultrasound-guided thoracic paravertebral block combined with general anesthesia. Guangdong Medical Journal 2019;40:1861-6.

17. Ono K, Danura T, Koyama Y, et al. Combined use of paravertebral block and general anesthesia for breast cancer surgery. Masui 2005;54:1273-6.

18. Yin YP, Wu P, Cui K, et al. Application of continuous thoracic paravertebral nerve block in general anesthesia for breast cancer. Chinese Journal of Practical Nervous Diseases 2019;22:406-12.

19. Ji XC. Effect of Continuous Ultrasound-guided Paravertebral Nerve Block on the Pain and Stress Response of Patients Undergoing Radical Mastectomy. The Chinese Journal of Burns Wounds and Surface Ulcers 2020;32:120-3.

20. Jin HX, Zhang TJ, Sun XF, et al. Effect of ultrasoundguided continuous thoracic paravertebral nerve block 
on chronic post-surgical pain in patients undergoing radical mastectomy for breast cancer. China Medicine 2018;13:1059-62.

21. Yang G, Liu ZY, Li G, et al. Effect of thoracic paravertebral nerve block on prevention of postoperative analgesia in breast cancerAbstract. Journal of Hunan Normal University (Medical Science) 2018;15:126-9.

22. Qian B, Lin Q, Chen TT, et al. Ultrasound-guided paravertebral blocks alleviate cellular immune dysfunction after modified radical mastectomy. Journal of Trauma and Emergency (Electronic Version) 2019;6:198-202.

23. Ding WP, Jia MX, Qiao L, et al. Application of Ultrasound-Guided Thoracic Paravertebral Nerve Block in Radical Mastectomy. Anti-Tumor Pharmacy 2019;9:641-5, 666.

24. Mayur N, Das A, Biswas H, et al. Effect of Clonidine as

Cite this article as: Feng C, Qian D, Chen C. A systematic review and meta-analysis of the effects of general anesthesia combined with continuous paravertebral block in breast cancer surgery and postoperative analgesia. Gland Surg 2021;10(5):1713-1725. doi:10.21037/gs-21-272
Adjuvant in Thoracic Paravertebral Block for Patients Undergoing Breast Cancer Surgery: A Prospective, Randomized, Placebo-controlled, Double-blind Study. Anesth Essays Res 2017;11:864-70.

25. Accurso A, Rocco N, Della Corte GA, et al. Breast Cancer Surgery Under General Anesthesia: Is it Really Worth it? Breast J 2017;23:492-4.

26. Tsigonis AM, Al-Hamadani M, Linebarger JH, et al. Are Cure Rates for Breast Cancer Improved by Local and Regional Anesthesia? Reg Anesth Pain Med 2016;41:339-347.

27. Maheshwari P, Maheshwari P. Single-injection vs continuous thoracic paravertebral block for postoperative analgesia after mastectomy. J Clin Anesth 2016;28:90-1.

(English Language Editor: A. Kassem) 